
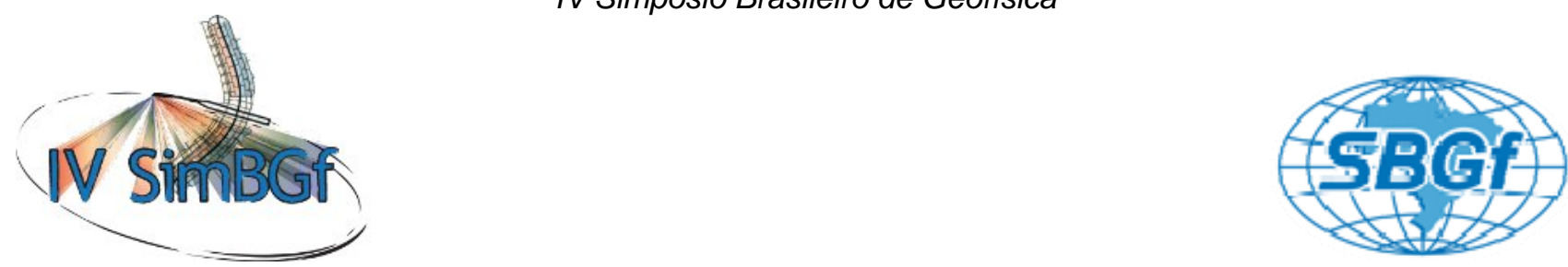

\title{
Utilização de Atributos da Onda Stoneley para determinação da Permeabilidade da Formação - Análise de Resultados.
}

Carlos Francisco Beneduzi, Petrobras

Copyright 2010, SBGf - Sociedade Brasileira de Geofísica No poo B (Figura 5), Kston e Kplug apresentam boa correlao apenas nos intervalos mais porosos do reservatrio (intvs, X250-X263m, X266-X274m, X290-X320m). Nos intervalos mais fechados, a correlao desaparece.

Este texto foi preparado para a apresentação no IV Simpósio Brasileiro de Geofísica, Brasília, 14 a 17 de novembro de 2010. Seu conteúdo foi revisado pelo Comitê Técnico do IV SimBGf, mas não necessariamente representa a opinião da SBGf ou de seus associados. É proibida a reprodução total ou parcial deste material para propósitos comerciais sem prévia autorização da SBGf.

\section{Resumo}

A permeabilidade da formação é um atributo petrofísico fundamental para monitorar a produção de um reservatório saturado de hidrocarboneto. Sua relação com a propagação e atenuação de uma onda acústica foi estudada exaustivamente nos últimos 30 anos.

A teoria da propagação da onda Stoneley no poço mostra que rochas permeáveis provocam atenuação na amplitude da onda e aumento na vagarosidade. Diversos algorítmos foram elaborados tentando relacionar a variação desses atributos com a permeabilidade. Alguns foram aproveitados pelas companhias de serviço que desenvolveram programas a partir de algumas dessas formulações. O trabalho analisa os resultados obtidos com diferentes programas e modelos aplicados em quatro poços perfurados em reservatórios siliciclásticos e carbonáticos. Os resultados foram comparados com outros registros de permeabilidade (NMR e dados de plugues) e mostram que é possível obter valores qualitativos em rochas siliciclásticas. Em carbonatos os resultados não se mostraram satisfatórios até 0 momento.

O trabalho é a apresentação de resultados iniciais e deve ser considerado com cautela. A análise de um maior número de dados permitirá conclusões mais definitivas.

\section{Introdução}

O cálculo da permeabilidade através da onda Stoneley baseia-se na troca hidráulica entre os sistemas de fluido presentes no poço e na formação. Por isso pode ser considerada uma medida direta da permeabilidade, a exemplo das medidas obtidas em testes de formação; ambas dependem da lei de Darcy. Além do mais é uma medida contínua e realizada in situ

A onda Stoneley é o modo principal de propagação de uma fonte monopolo; propaga-se ao longo da interface poço-formação (onda de superfície) comprimindo o fluido do poço para a formação, com um efeito similar ao de um pistão. Frente a uma rocha permeável ocorre um deslocamento de fluido do poço para a formação e conseqüente perda de energia (atenuação) e redução de velocidade da onda. (Fig. 1)

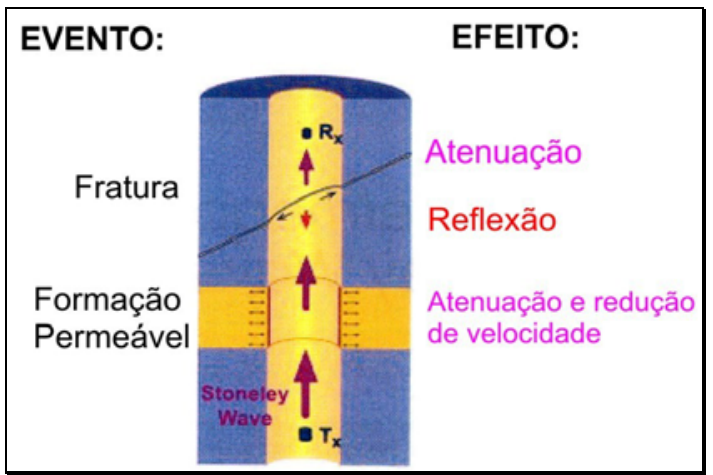

Fig. 1 - Efeito produzido por permeabilidade e fraturas na propagação da onda Stoneley (Adaptado)) de Brie, A. et al. (2000

Esse fluxo do poço para a formação, provocado por um gradiente de pressão mede, na realidade, a mobilidade (permeabilidade/viscosidade) do fluido.

A grande dificuldade enfrentada por todos os modelos é separar os efeitos provocados pela permeabilidade daqueles provocados por outras causas: atenuação intrínseca da formação, elasticidade da formação, irregularidades no diâmetro do poço e mudança de formação, efeito do reboco, anisotropia. Outro fator complicador para o estabelecimento de valores absolutos é o fato da investigação ser feita predominantemente na zona invadida (profundidade de investigação- $0.5 / 1$ pé; resolução vertical - 3.5 pés) o que exige determinação correta dos parâmetros elásticos dos fluidos envolvidos, em especial no caso da presença de gás.

\section{Fundamentos Teóricos e Definições}

De acordo com Biot (Biot, 1956a, b,1962) três tipos de ondas propagam-se em um meio sólido poroso saturado por um fluido: onda compressional rápida, onda cisalhante e onda compressional lenta. As ondas rápidas estão associadas principalmente com o movimento da matriz sólida, modificada pela presença de fluido nos poros; as ondas lentas associam-se primariamente com o movimento do fluido nos poros, modificado pela presença de matriz sólida. O mecanismo de excitação e propagação das ondas lentas no fluido que preenche os poros da rocha é a base para a estimativa da permeabilidade da formação através da onda Stoneley.

A propagação da onda Stoneley no poço excita os três tipos de onda na formação. A teoria de Biot-Rosembaum 
trata da interação entre a onda de poço e os três tipos de onda gerados na formação utilizando a equação de Biot para meios poroelásticos. Apesar de acurado, o método envolve cálculos complexos que demandam extenso tempo de computação.

Como alternativa foi elaborado um modelo simplificado (Tang et al.,1991a). Nele decompõe-se o processo de excitação da parede permeável de um poço por uma onda Stoneley em duas contribuições ( Figura 2):

-o deslocamento elástico da parede, que remete ao problema de um poço constituido por uma for-mação elástica composta por um esqueleto poroso saturado por fluido (apenas ondas P e S são consideradas na solução análogas à compressional rápida e cisalhante da Teoria de Biot)

-fluxo de fluido através dos poros que remete ao problema relacionado ao mecanismo de propagagação da compressional lenta da Teoria de Biot responsável pela dissipação da energia da onda Stoneley para a formação; principal fenômeno responsável pela efeito da permeabilidade sobre atenuação e velocidade da onda Stoneley.

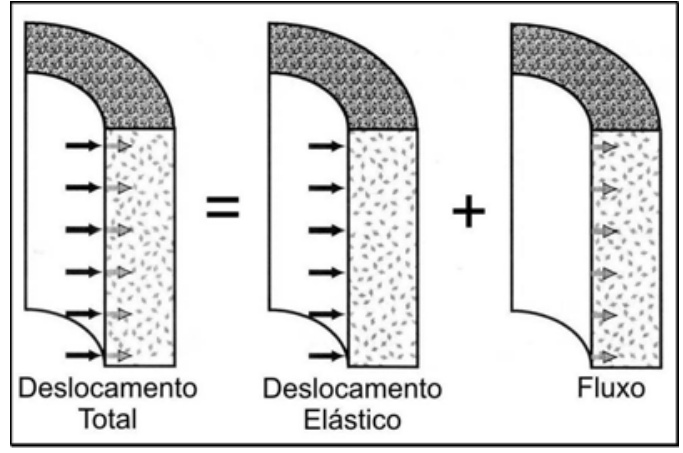

Fig. 2 -A oscilação da parede de um poço pro-vocada pela passagem de uma onda Stoneley resulta da interação entre deformação elástica da rocha e fluxo de fluido pela parede do poço. (Adaptado de Tang, X.$M$ et al., 2004)

Os resultados de ambos os métodos, mostraram-se muito próximos, após algumas correções em discrepâncias observadas em formações pouco consolidadas resultando na equação abaixo para a propagação da onda Stoneley em rochas permeáveis atravessadas por um poço.

$$
k=\sqrt{k_{e}^{2}+\frac{2 i \rho_{p f} \omega \mathrm{k}(\omega) R}{\eta\left(R^{2}-a^{2}\right)} \frac{\sqrt{-i \omega / D+k_{e}^{2}}}{1+B C^{V s / V f}} \frac{K_{1}\left(R \sqrt{-i \omega / D+k_{e}^{2}}\right)}{K_{0}\left(R{\sqrt{-i \omega / D+k_{e}}}^{2}\right.}}(1)
$$

onde:

k é o número da onda Stoneley, $k_{e}$ é o número da onda Stoneley elástica, $\rho_{p f}$ é densidade do fluido da formação, $\kappa(\omega)$ é a permeabilidade dinâmica, $R$ é o raio do poço, $D$ é a difusividade do fluido, $\eta$ é viscosidade do fluido , a é o raio da ferramenta, $\omega$ é a frequência angular, $\quad K_{0}$ e $K_{1}$ são os módulos da incompressibilidade do meio, $V s$ é a velocidade cisalhante, $V f$ é velocidade de fluido no poço. $B C$ (maleabilidade do poço) é definida por:

$$
B C=f_{e} R \frac{I_{1}\left(f_{e} R\right)}{I_{0}\left(f_{e} R\right)}
$$

com $f_{e}=\left(k_{e}^{2}-k_{f}^{2}\right)^{1 / 2}$ (número da Onda Stoneley radial)

e $k_{f}$, módulo da incompressibilidade do fluido presente no poço

\section{Estimativa de permeabilidade a partir de ondas Stoneley geradas no poço}

No ambiente de poço, para o caso do transmissor monopolo, são levados em consideração três tipos de onda na formação: onda compressional rápida, onda compressional lenta e onda cisalhante (tipo SV) com características de propagação semelhantes às modeladas na teoria de Biot-Rosembaum. Pode-se medir diretamente através de uma ferramenta acústica a compressional rápida e a cisalhante. Entretanto, a compressional lenta não pode ser obtida pois não é refratada dado que sua velocidade é inferior à velocidade do fluido presente no poço. Seu efeito se manifesta através da comunicação de pressão entre o fluido presente no poço com o fluido presente nos poros da formação. A onda Stoneley, onda de pressão de baixa freqüência originada no fluido presente no poço, é sensível a essa comunicação de pressão na interface do poço sendo usada para relacionar uma medida acústica de poço e as propriedades de transporte do fluido da formação.

Diversos métodos foram desenvolvidos para obter a permeabilidade a partir de atributos da onda Stoneley:

a. Através da vagarosidade da onda Stoneley .

Hornby (1989) observou que a propagação da onda Stoneley dentro de um poço em uma rocha poropermeável afetava sua vagarosidade e que esta era afetada pela elasticidade da formação e propriedades dos fluidos presentes no poço. Isso foi traduzido na equação (aproximação da equação (1) com $\omega \rightarrow 0$ e a=0) :

$$
S^{2}=\left(\frac{\rho_{f}}{K_{f}}+\frac{\rho_{f}}{\mu}\right)_{\text {elást }}+\left(\frac{2 \rho_{f} i k_{0}}{\eta \omega R} \sqrt{-i \omega / D} \frac{K_{1}(R \sqrt{-i \omega / D})}{K_{0}(R \sqrt{-i \omega / D})}\right)_{\text {fluxc }}
$$

onde a vagarosidade da Stoneley (S) é controlada pelo módulo de cisalhamento da formação $(\mu)$, pelo módulo da incompressibilidade do fluido presente no poço $\left(K_{f}\right)$, densidade do fluido presente no poço $\left(\rho_{f}\right)$ e pelas propriedades do fluxo na formação através da difusividade do fluido $(D)$.

A permeabilidade é resultado da diferença entre vagarosidade medida da onda Stoneley e a vagarosidade 
da onda Stoneley elástica, obtida da vagarosidade da onda cisalhante e da vagarosidade do fluido presente no poço.

O problema dessa formulação é que o segundo termo da equação apresenta valores muito pequenos comparados aos do primeiro e incertezas relativas aos módulos de cisalhamento da formação e bulk do fluido podem ser da mesma magnitude e até maiores que os efeitos da permeabilidade tornando difícil a distinção entre estes e os efeitos de não-permeabilidade.

\section{b. Através da amplitude da onda Stoneley}

Modelo proposto por Tang e Cheng (1996) a partir da constatação do decaimento da amplitude da onda Stoneley observado em rochas permeáveis. O espectro de amplitude da onda Stoneley em um poço permeável pode ser descrito como:

$$
A(\omega, z)=S R(\omega) E(\omega, z) \exp \left(-\frac{\omega d}{2 Q V_{S T}}\right)
$$

onde $S R(\omega)$ representa a fonte e o espectro de resposta do receptor; $d$ a distância fonte-receptor; $z$ a profundidade de referência; $Q^{-1}$ e $V_{S T}$, atenuação e velocidade da onda Stoneley; $E(\omega, z)$ função excitação da onda Stoneley, que pode ser calculada da excitação da formação elástica equivalente $E_{e}(\omega, z)$.

A partir de uma referência impermeável em $\mathrm{z}_{0}\left(\mathrm{Q}^{-1}=0\right)$ a permeabilidade da formação pode ser calculada em $z_{1}$ através da minimização da função objetiva:

$$
\left.\left.O b\left[k_{0}(z)\right]=\int_{\omega}\left\{A\left(\omega, z_{0}\right) \exp -\frac{\omega d}{2 Q\left(k_{0}\right) V_{S T}\left(k_{0}\right)}\right)-\left|\frac{k(z) E_{e}\left(\omega, z_{0}\right)}{k(z) E_{e}(\omega, z)}\right| A(\omega, z)\right)\right\}^{2} d \omega
$$

O método funciona para valores de permeabilidade altos (da ordem de darcys). Para valores de permeabilidade médios ou baixos, mudanças de amplitude causadas por outros efeitos (atenuação intrínseca, mudanças de formação e diâmetro de poço) podem obscurecer os causados pelas mudanças de permeabilidade.

c. Através da amplitude e fase da onda Stoneley

O modelo que propõe um valor estimado de permeabilidade consta de três etapas:

1. Separação do trem de ondas Stoneley em diretas, ascendentes e descendentes para suprimir os efeitos causados por ruído de viagem, espalhamento e reflexão provocadas pelas irregularidades do poço e mudanças de formação.

2. Modelagem da amplitude e mudança de fase da onda Stoneley através do método da matriz de propagação (Tang,(1996) e Tezuka et al.(1997)), levando em consideração as irregularidades do diâmetro do poço e as mudanças das propriedades elásticas das formações atravessadas pelo poço.

3. Obtenção de um indicativo de permeabilidade através da diferença entre valores medidos de atenuação (frequency shift) e velocidade da onda (travel-time delay) (item 1), dos valores obtidos através de modelagem numérica (item 2) (Fig. 3).

$$
\begin{aligned}
& \Delta f_{C}=f_{C}^{\text {syn }}-f_{C}^{\text {msd }} \\
& \Delta T_{C}=T_{C}^{\text {msd }}-T_{C}^{\text {syn }}
\end{aligned}
$$

Para um dado espectro de onda (sintético ou medido) a frequência central e sua variância são representadas por:

$$
\begin{aligned}
f_{C} & =\int f W(f) d f / \int W(f) d f \\
\sigma^{2} & =\int\left(f-f_{C}\right)^{2} W(f) d f / \int W(f) d f
\end{aligned}
$$

O tempo central é obtido da forma de onda (W) através da fórmula:

$$
T_{C}=\int t(W(t))^{2} d t / \int(W(t))^{2} d t
$$

A atenuação devido à formação e ao fluido presente no poço também é levada em consideração através da fórmula elaborada para essa finalidade.

A partir do modelo teórico calcula-se a atenuação da onda (frequency shift) teórica:

$$
\Delta f_{C}^{\text {theo }}=f_{C}^{\text {syn }}-f_{C}^{\text {theo }}
$$

com freqüência central e variância dadas por:

$$
\begin{aligned}
f_{C}^{\text {theo }} & =\int f P W^{\text {syn }}(f)\left|e^{i k d}\right| d f / \int P W^{s y n}(f)\left|e^{i k d}\right| d f \\
\sigma_{\text {theo }}^{2} & =\int\left(f-f_{C}^{\text {theo }}\right)^{2} P W^{\text {syn }}(f)\left|e^{i k d}\right| d f / \int P W \quad(f)\left|e^{i k d}\right| d f
\end{aligned}
$$

Com $P=k_{e} / k$ (redução de excitação) e $\left|e^{i k d}\right|$ (decaimento de amplitude) provocados pela permeabilidade.

A redução da velocidade teórica é calculada no domínio da frequência:

$$
\Delta T_{C}^{\text {theo }}=\int\left(k d / \omega-k_{e} d / \omega\right)\left(\omega W^{\text {syn }}(f)\right)^{2} d f / \int\left(\omega W^{\text {syn }}(f)\right)^{2} d f
$$

Da comparação entre as equações 6, 9 e 11 formula-se um problema inverso cujo resultado é minimização da função objetiva:

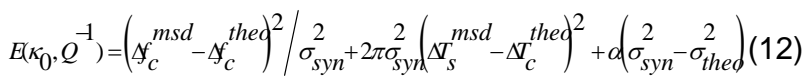

em que $\sigma_{\text {syn }}^{2}$ e $\sigma_{\text {theo }}^{2}$ são obtidos das equações 7 e 10.

A função objetiva é a soma dos dados de freqüência e tempo (equações 7 e 8 ) registrando a permeabilidade e/ou os efeitos da atenuação nos dados de freqüência e tempo de trânsito.

O valor absoluto de permeabilidade depende de valores de viscosidade $(\eta)$, densidade e velocidade dos fluidos 
$\left(\rho_{\mathrm{pf}}\right.$ e $\mathrm{V}_{\mathrm{pf}}$ ) presentes nos poros, combinados na expressão:

$\frac{\kappa_{0}}{\eta K_{p f}^{1 / 2}},\left(K_{p f}=\rho_{p f} V_{p f}^{2}\right)$

São valores difíceis de serem obtidos no caso de diversos fluidos saturarem a rocha. Para sua determinação, se faz necessária uma calibração pelo menos em duas profundidades com valores de permeabilidade de outras medidas (NMR, testemunho, WFT). O termo $\eta K_{p f}^{1 / 2}$ é estimado através da minimização de uma função de ajuste para cada profundidade e permite a obtenção de uma curva de permeabilidade para todo o intervalo de interesse.

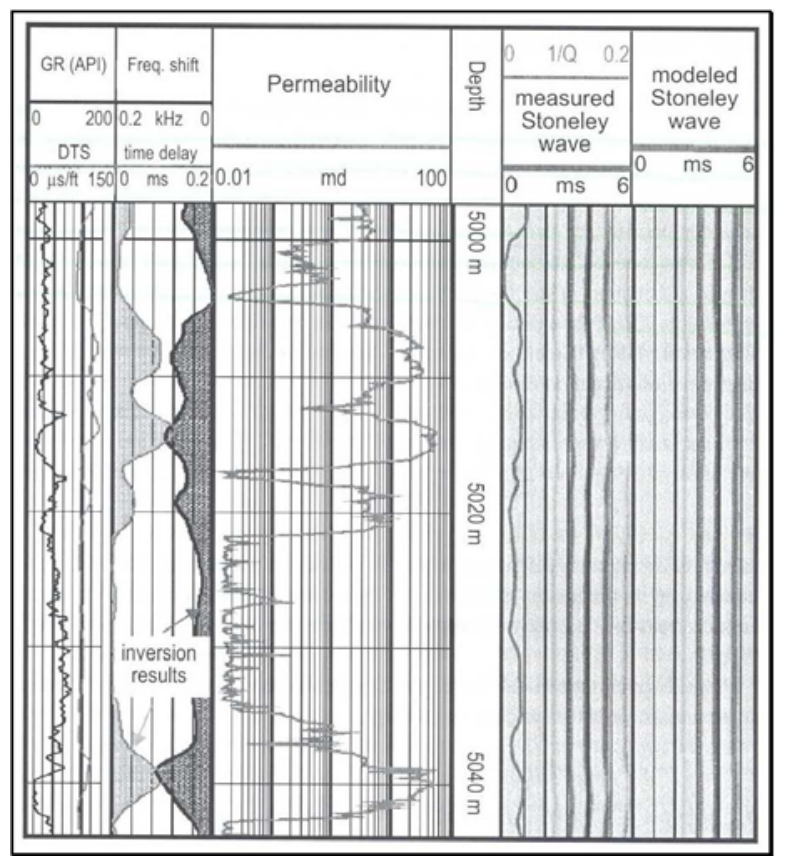

Fig. 3 - Resultado do processamento mostrando as anomalias nas curvas Frequency Shift e Time Delay (track 2) correspondentes a anomalias na curva de permeabilidade (track 3).(Tang, X.-M et al., 2004)

d. Através da vagarosidade e atenuação da onda Stoneley em uma análise multifrequência.

A inversão é o resultado da integração do modelo poroelástico de Biot de propagação da onda Stoneley ao qual é adicionado o efeito do reboco considerado como membrana elástica, implementado por Liu e Johnson (1997) com técnicas estatísticas de processamento e análise de erro desenvolvidas por Kimball (1995). A análise de erro permite quantificar o efeito da incerteza nos parâmetros envolvidos no modelo e o erro total do valor de mobilidade obtido.

O poço é modelado com uma camada elástica e flexível (reboco) interposta entre o fluido de perfuração e a rocha da formação. Uma onda oscilatória de pressão produz um fluxo de entrada e saída no meio poroso ocasionando atenuação e dispersão da onda. A flexibilidade do reboco é introduzida adicionando-se uma membrana rígida na parede do poço que permita, à semelhança dele, movimentar-se na parede do poço de acordo com a direção do fluxo. O mecanismo reduz sem eliminar os efeitos da permeabilidade na onda Stoneley. (Fig. 5) O modelo propõe-se a calcular mobilidade (permeabilidade/viscosidade), pois a ação da onda Stoneley sobre uma rocha permo-porosa provoca um fluxo com um diferencial de pressão constante, definição de mobilidade.

A dependência da mobilidade à vagarosidade pode ser visualizada na figura 6 e revela a importância da escolha criteriosa dos valores para os parâmetros de processamento. A análise de erro mostra que $90 \%$ provém de 3 parâmetros ( vagarosidade e atenuação do fluido de perfuração e módulo bulk do fluido presente nos poros).

\section{Exemplos/Resultados}

Foram testados dois pacotes de processamento de diferentes companhias de serviço. Em nenhum deles foram calculados valores absolutos, embora ambos os programas possuam rotinas com essa finalidade.

\section{Companhia A}

O pacote é desenvolvido sobre o modelo que estima a permeabilidade analisando o comportamento de amplitude e fase da onda Stoneley.

Foram analisados registros em reservatórios siliciclásticos de baixa permeabilidade com porosidades efetivas entre 15-20\% (poços A e B), e em reservatório carbonático com porosidades entre $12-15 \%$ (poço C). Em todos os exemplos houve comparação entre as medidas de plugues de laboratório, com dados de NMR, onde a permeabilidade foi obtida, nos poços $A$ e $B$ através da calibração dos coeficientes da equação de Timur-Coates com os plugues, e no poço $\mathrm{C}$ com o valor de cutoff défault para carbonatos.

No poço A (Figura 4), observa-se boa correlação com os valores de permeabilidade obtidos com Stoneley (Kston) com aqueles obtidos em laboratório (Kplug), inclusive em termos absolutos (intv. X987-X000m). No intv X020X050m há um descolamento nos valores porém a tendência de diminuição dos valores é mantida em ambas as medidas.

No poço B (Figura 5), Kston e Kplug apresentam boa correlação apenas nos intervalos mais porosos do reservatório (intvs X250-X263m, X266-X274m, X290$\mathrm{X} 320 \mathrm{~m})$. Nos intervalos mais fechados, a correlação desaparece.

No poço C (Figura 6), a análise dos dados não afetados pelas más condições de poço (abaixo de X070m) mostra que à exceção dos pontos de maior porosidade (X080X083m) não há correlação entre Kston e Kplug. A melhor correlação se dá entre Knmr e Kplug. A nãocorrespondência de comportamento entre Kston e as 
curvas de travel time delay e frequency shift (track 4) sugere que possa haver algum problema de processamento.

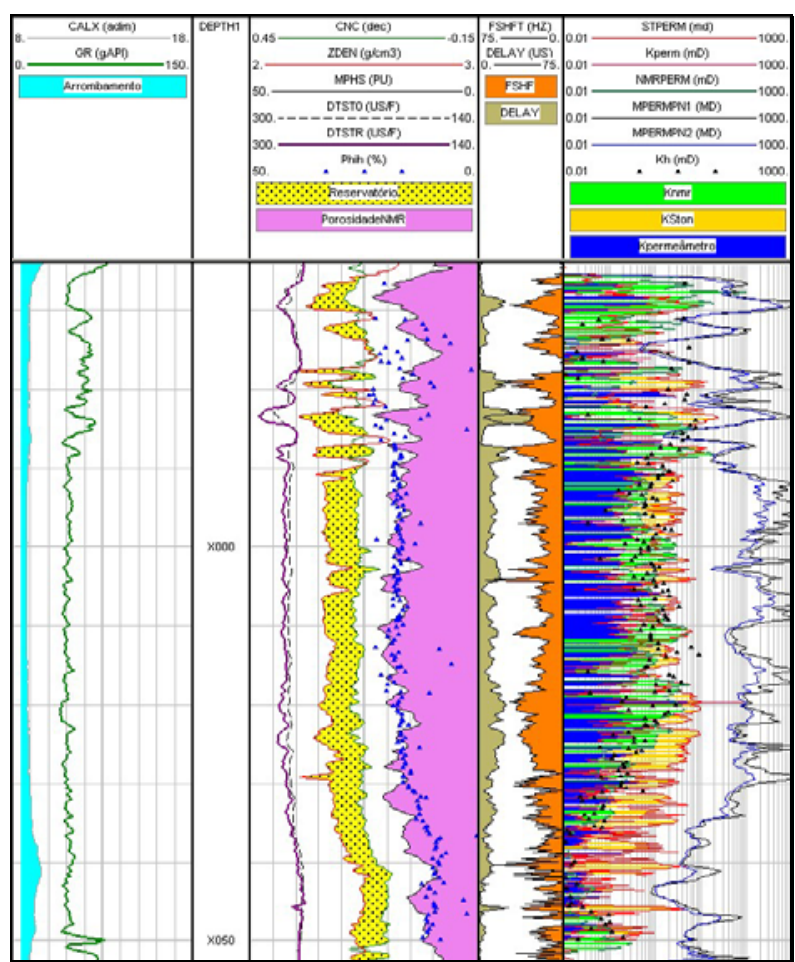

Fig. 4 -No poço A observa-se boa correlação entre Knmr(verde) e dados de plug (triângulos azuis). Dados de permeâmetro (azul) são mais pessimistas.e valores de Kston (amarelo) ligeiramente otimistas.

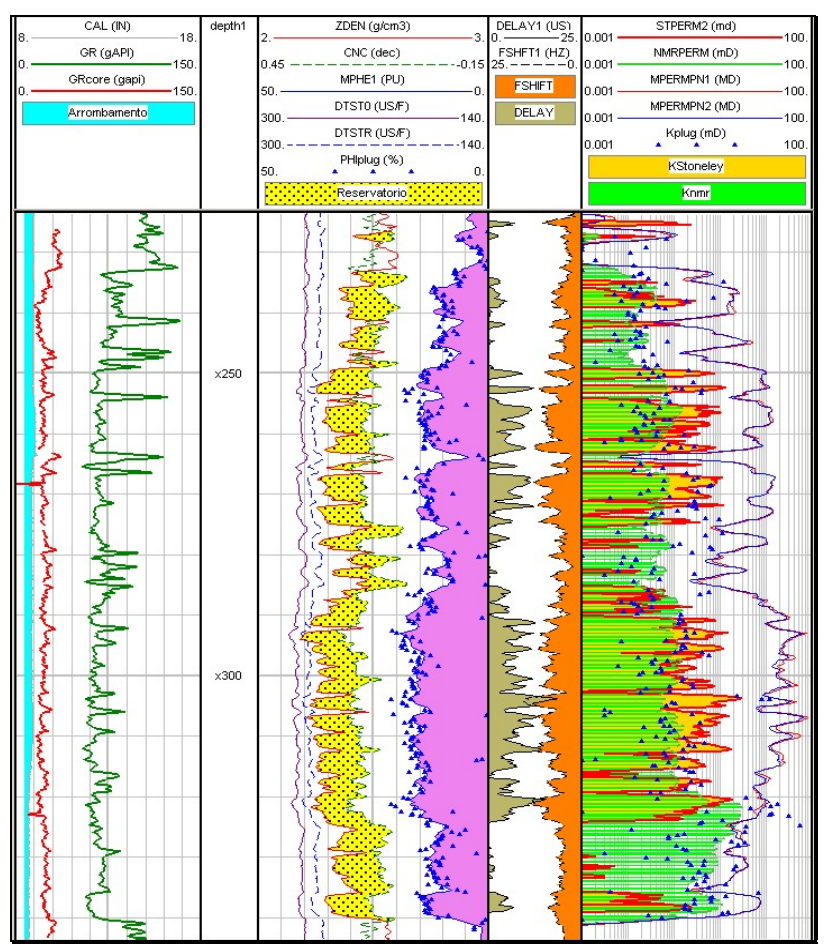

Fig. 5 - No poço B, observa-se boa correlação entre Kston e Kplug nos intvs mais porosos (intv. X250$X 263 m, X 266-X 274 m, X 290-X 320 m)$. A melhor correlação se faz com Knmr calibrado(verde).

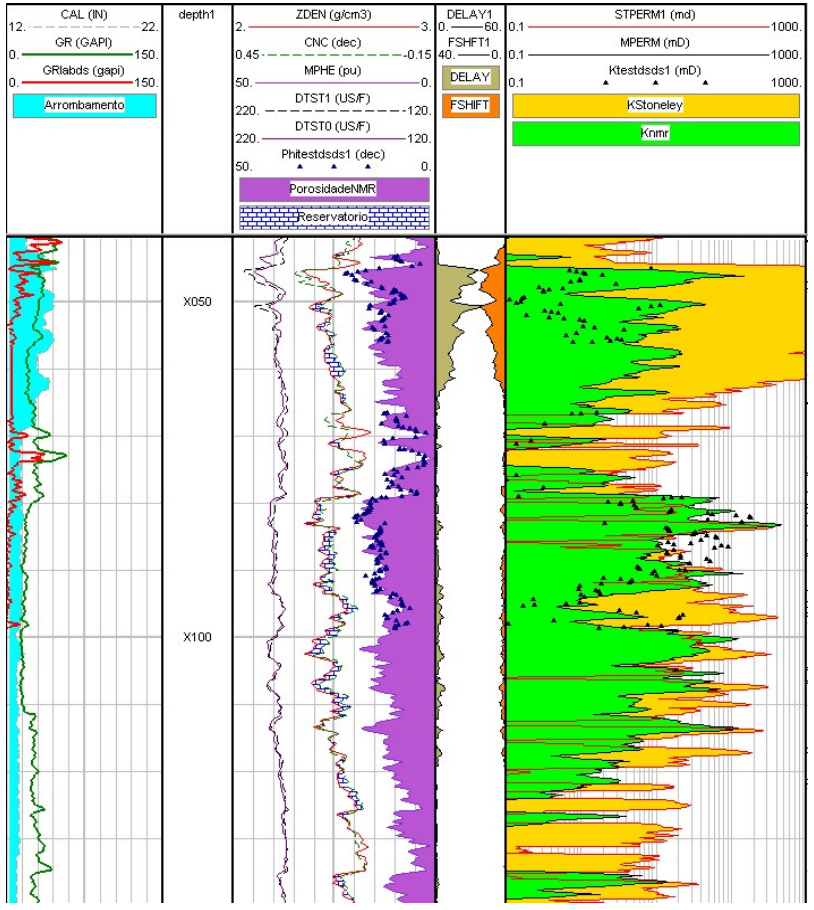

Fig. 6 - No poço C (carbonatos), as condições de caliper impossibilitam a análise até X070m. Abaixo, a correlação de Kplug é melhor com Knmr; com Kston, apenas no intv X080-X083m.

\section{Companhia B}

Possue um pacote de processamento que engloba 3 modelos para obtenção de mobilidade do fluido na formação: análise de vagarosidade da onda Stoneley em baixa freqüência, de atenuação da onda Stoneley em baixa freqüência e análise multifrequência de vagarosidade e atenuação da onda Stoneley.

Foi analisado, através do último método, apenas um registro em reservatório carbonático (poço D) (Figura 7) com porosidades entre 8 e 12\% (o método é aplicável para porosidades superiores a 10\%). O valor de mobilidade foi confrontado com dados de Knmr ( valores de permeabilidade obtidos com equação de TimurCoates utilizando valores de cutoff défault) e Kplug. Apresentou boa correlação com o primeiro, mesmo em baixas porosidades. Ambos, entretanto, mostram-se otimistas em relação aos valores de laboratório com discrepâncias na ordem de um ciclo logarítmico.

No único registro em siliclásticos que dispunha de dados de laboratório e que se enquadrava nas condições de contorno estabelecidas para o método os resultados obtidos não foram satisfatórios. Continua-se trabalhando em novos poços 


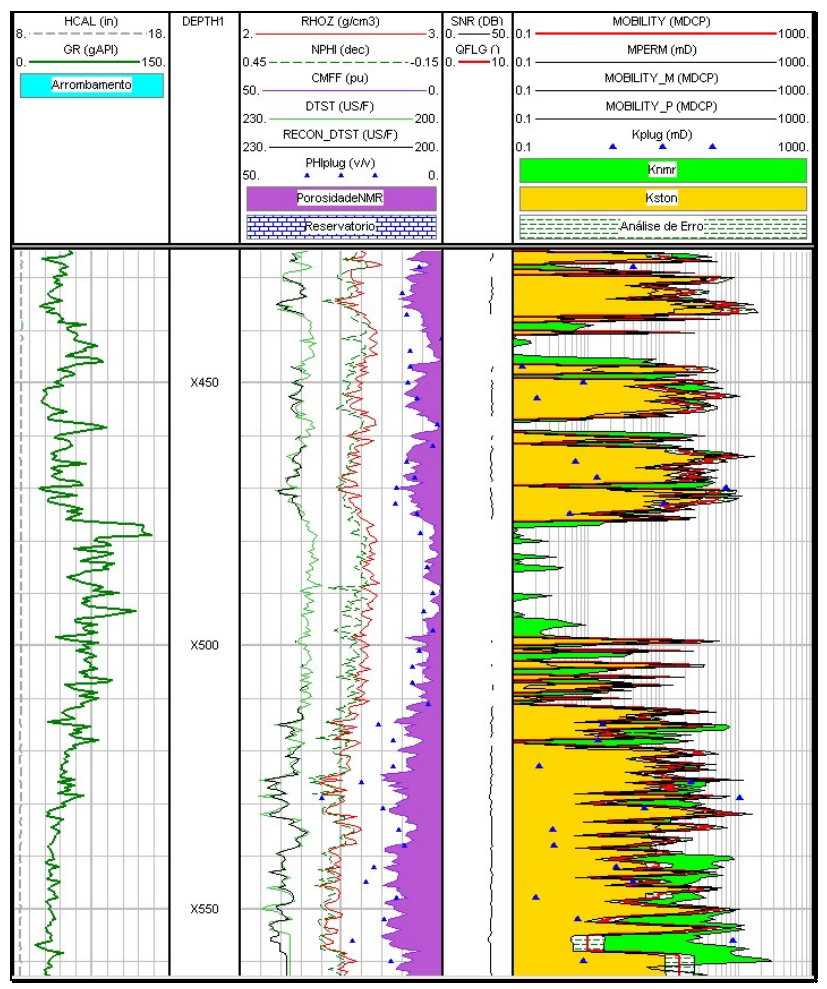

Fig. 7 - No poço D, Kston e Knmr apresentam boa correlação entretanto ambos divergem em relação a Kplug. O comportamento mantém-se inalterado mesmo em porosidades abaixo de $10 \%$.

\section{Conclusões}

O pequeno número de poços analisados não permite conclusões definitivas sobre os pacotes de processamento. Todos os poços apresentaram baixas porosidades e permeabilidades, fato que dificulta a obtenção de valores confiáveis especialmente no pacote de processamento da companhia $B$.

É importante que o processamento seja feito por técnicos qualificados em perfis acústicos, pois a confiabilidade do produto final depende muito da escolha criteriosa de alguns parâmetros de processamento.

Dos dados analisados, pode-se afirmar que em rochas siliciclásticas o método apresentou valores mais próximos aos de laboratório. No pacote da empresa $A$, intervalos de menor porosidade não apresentaram boa resposta. Em carbonatos, entretanto, os dados não se apresentam ainda confiáveis.

Com o atual aumento na amostragem lateral de poços, abre-se a oportunidade de, a partir das medidas de laboratório cotejar e calibrar melhor os dados obtidos através de perfis e assim dar confiabilidade ao método e torná-lo mais uma ferramenta disponível na determinação da permeabilidade do reservatório.

\section{Agradecimentos}

Ao engenheiro da Baker-Atlas, Gustavo Jesus Pérez pelo processamento dos poços cujos dados foram adquiridos pela companhia. Aos geólogos Sandra Reveriego Carneiro e Gabriel Luiz Pérez-Vieira pelos dados calibrados de $K n m r$ dos poços A e B; ao engenheiro Vinicius de França Machado pelos dados de laboratório; ao geofísico Elton Luiz Diniz Ferreira pelas discussões a respeito dos aspectos físicos e matemáticos.

\section{Referências}

Brie, A., Endo,T., Johnson, D.L., and Pampuri, F., 2000, Quantitative Formation Permeability Evaluation from Stoneley Waves, SPE 60905.

Biot, M. A., 1956a, Theory of propagation of elastic waves in a fluid-saturated porous solid, I: Low Frequency Range: J. Acoust. Soc. Am., 28, 168-178.

Biot, M. A., 1956a, Theory of propagation of elastic waves in a fluid-saturated porous solid, II: High Frequency Range: J. Acoust. Soc. Am., 28, 179-181.

Biot, M. A., 1962, Mechanics of deformation and acoustic wave propagation in porous media : J. Appl. Phys., 33, 1482-1498.

Hornby, B.E., 1989, Method for determining formation permeability by comparing measured tube waves with formation and borehole parameters, U.S Patent 4.789.859.

Kimball, C.V., Lewicki. P. and Wijeyesekera, N., 1995, Error analysis of maximum likelihood estimates of physical parameters from one or more dispersive waves: IEEE Transactions and Signal Processing, 43, 29282936.

Liu,H.,-L., and Johnson, D.L.,1997, Effects of an elastic membrane on tube waves in permeable formations: J. Acoust. Soc. Am., 101, 3322-3329.

Pampuri, F., Rovellini, M., Brie, A., Fukusima, T., 1998, Effective Evaluation of Fluid Mobility From Stoneley Waves Using Full Biot Model Inversion: Two Case Histories, SPE 49132

Tang, X.M., 1996, Fracture Hydraulic Conductivity Estimation from Borehole Stoneley Wave Transmission and Reflection Data, paper $\mathrm{HH}, 37^{\mathrm{a}}$ SPWLA

Tang, X.M, Cheng, A., 2004, Quantitative Borehole Acoustic Methods. Amsterdam, Elsevier, 255 p.

Tang, X.,M., Cheng, C., H., 1996, Fast Inversion of Formation Permeability from Stoneley Wave Logs Using a Simplified Biot-Rosembaum Model, Geophysics, 61, 639645.

Tang, X.,M., Cheng, C., H., and Toksoz, M.N., 1991a, Dynamic Permeability and Borehole Stoneley Waves:A simplified Biot-Rosembaum Model: J. Acoust. Soc. Am., 90, 1632-1646.

Tezuka, K., Cheng, C.H.,Tang, X.M., 1997, Modeling of Low Frequency Stoneley-wave Propagation in an Irregular Borehole: Geophysics, 62, 1047-1058.

Winkler, K.W., Liu, H.-L., and Johnson, D.L.,1989, Permeability and borehole Stoneley waves: Comparison between experiment and theory: Geophysics, 54, 66-75. 